\title{
Perceived militating factors to the practice of preventive measures for COVID-19 pandemic among health professionals in public health facilities of southern Ethiopia: a phenomenological study
}

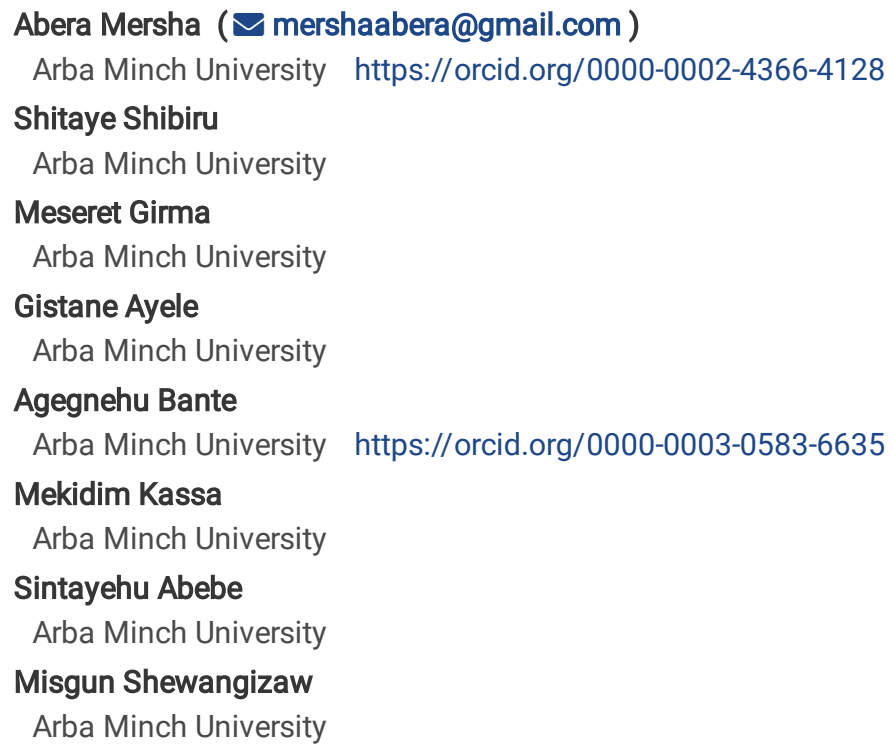

\section{Research Article}

Keywords: Coronavirus, pandemic, militating factors, phenomenological study, Ethiopia

Posted Date: October 20th, 2020

DOI: https://doi.org/10.21203/rs.3.rs-95576/v1

License: (c) (i) This work is licensed under a Creative Commons Attribution 4.0 International License. Read Full License 


\section{Abstract}

Background: Novel coronavirus is a global pandemic and killed many individuals, including health care professionals. It caused stress on the health care system of all countries. Presently, studies are emerging regarding the COVID-19 pandemic in different aspects. However, a few have explored militating factors that affecting the practice of preventive measures for the COVID-19. As such, the study aimed to fill these research gaps in the study setting.

Methods: A semi-structured interview guide used to conduct this phenomenological study among 16 key informants. Key informants recruited by the purposive sampling method. The data analyzed inductively by using a thematic content analysis approach in NVivo 12 Pro software.

Results: In this study, six main themes identified with the sub-themes. Overview of COVID-19 pandemic, consequences (with the two subtheme), perceived practice (with four sub-theme), perceived militating factors (with four sub-theme), newfangled activities (with three subtheme), and suggestion for improvement (with seven sub-theme) were the major themes. The participants perceived the influence of shortage of personal protective equipment and solutions for hand hygiene, negligence and ignorance, inadequate infrastructure, lack of training, and lack of attention and recognition for the staff on the practice of preventive measures.

Conclusions: Conclusions: This study showed that there were gaps in the practice of preventive measures for the COVID-19 among health professionals in the health care system. Community influences, health care provider related factors, institutional factors, and lack of communication and support affect the practice. Hence, attention should give to fulfill the necessary supplies in the health facilities, improve the infrastructures, and equip health professionals by providing capacity-building activities. Besides, health care professionals must recognize, and credit should consider.

\section{Background}

Coronavirus disease-2019 (COVID-19) is a highly contagious acute respiratory disease. The causative agent is a novel coronavirus (2019-nCoV) and first evident in Wuhan City, Hubei province, China [1-6]. The known mode of transmissions are droplets, contact, and aerosol [7, 8]. The complete clinical manifestation is not clear yet, but the most commonly reported symptoms are fever, cough, myalgia or fatigue, pneumonia, and complicated dyspnea [9-11]. Real-time fluorescence (RT-PCR) detects the nucleic acid of SARS-CoV-2 [8, 12, 13].

Health care professionals (HCPs) are at the front line of the COVID-19 outbreak response [14, 15]. As such, contact and droplet precautions should practice. Health care professionals must use personal protective equipment (PPE) such as a glove, gown or apron, and surgical mask [16-18].

Globally, Coronavirus disease-2019 disrupts health care systems. It is crucial to protect those most impacted by COVID-19, sustain gains made to address other infectious diseases, and maintain people's access to life-saving health services [19]. The contained outbreak could significantly influence the global economy in the short run. The costs reduce by investing in public health systems in all countries. But, the focus should give for less developed countries where health care systems are weak, and population density is high [20]. The public health systems in Africa are coming under severe strain as the unprecedented COVID-19 pandemic persists [21, 22].

A qualitative study from China indicated that health care providers volunteered and tried their best to provide care for patients. They challenged by working in a very new context, exhaustion due to heavy workloads and protective gear, the fear of becoming infected and infecting others, feeling powerless to handle patients' conditions, and managing relationships in this stressful situation [23]. Several factors may influence the availability of health workers to deliver essential services, including redeployment of staff to treat increasing numbers of patients with COVID19 and the absence of health workers in quarantine. The combination of increased workload and reduced numbers of health workers is likely to pose a severe strain on the capacity to maintain essential health care services[24].

Infection control interventions to reduce transmission of COVID-19 include universal source control, early identification, and isolation of patients with suspected disease, the use of appropriate PPE when caring for patients with COVID-19, and environmental disinfection are obligatory in the health care settings[25]. The study by Khader et al. showed that $74.7 \%$ of study participants believed that it was necessary to ask patients to sit far from each other, wear masks while in the waiting room, and wash hands before getting in the dental chair to decrease disease transmission[26]. Findings from Pakistan also stated that $13.8 \%$ of participants remove the mask while talking to the patient, $20.2 \%$ reused, $44.9 \%$ correctly used the yellow-coded bag for disposal, $93.9 \%$ wear masks in clinics, and $94.6 \%$ wear masks in hospital premises [27]. A study identified that overcrowding, limited infection control material, less commitment of HCPs to the policies and procedures, insufficient training, and lack of policy and procedures of infection control practice were militating factors [28].

Presently, studies are emerging regarding the COVID-19 pandemic in different aspects. However, a few have explored militating factors that affecting the practice of preventive measures for the COVID-19 among health professionals. Therefore, there was a need to fill these research gaps in the study setting. 


\section{Methods}

\section{Study setting, design and period}

In this phenomenological study, key informants from the public health facilities of the southern Ethiopia, were involved, from June $10-30,2020$.

\section{Sampling}

A purposive sampling method was employed to recruit key informants. Chief executive officers, medical directors of the hospitals, health center heads, environmental health workers, chief nursing director, matron, heads in each ward or unit, and case team leaders involved for the key informants' interview (KII). In this study, sixteen key informants were involved, based on idea saturation.

\section{Data collection method}

Two interviewers were involved, and the information was collected using a semi-structured interview guide. The interview guide developed based on expert opinion, knowledge, and skill of investigators and existing works of literature (Table 1) (Supplementary file 1). Interviewers trained, and key informants, advertised in each participating health facility to involve in the study. The aim of the study, purposes, risks, and possible benefits, the right and refusal to participate in the study, confidentiality issues were provided for those who zealous to the participant. Then, to meet the study objective, pillar participants were purposively selected by the interviewer and involved in the KII. At the outset, the sociodemographic characteristics of each participant documented after getting written and signed consent. Each session of the KIls recorded by an audiotape recorder, and keynotes took. The interview conducted in one private room, which comfortable for the participants.

Table 1: Major points of the interview script

\begin{tabular}{|l|}
\hline Topics in the interview script \\
\hline What do you think about COVID-19? \\
\hline How health professionals prevent themselves and patients from COVID-19? \\
\hline What do you think about precautionary measures? \\
\hline What are the perceived militating factors for the practice of precautionary measures? \\
\hline What activities done in the health facility to prevent the spread of COVID-19? \\
\hline What do you suggest to improve the practice of health care providers to tackle the COVID-19 pandemic? \\
\hline
\end{tabular}

\section{Data quality control (trustworthiness)}

Trained and experienced interviewers collected the information. The interviewers communicated and discussed the points that they faced during the data collection period on a daily bases. The well-established, expert commented, reviewed semi-structured interview guide used to collect the data. The information sought from participants with relevant expertise and experience, a neutral view of the investigator maintained, and participants probed, and who had much on the issue selected to ensure trustworthiness. The participant's variations to emerge different ideas and experiences managed by involving Kls with different positions and responsibilities in the health facilities, and original audios and written transcripts re-checked to develop themes to achieve data credibility. To achieve conformability, an NVivo 12 Pro software used to enhance the maintenance of connections between participant descriptions and the interpretation or the synthesis researcher made. The result presented alongside direct quotes from participants. The recorded interview transcripts, translations, and demographic summary of participants were maintained, and keynotes took during and after (during analysis) the interview to achieve the dependability of standards.

\section{Data analysis}

The audios of each interview that transcribed verbatim in the local language independently heard more times by the two investigators until they familiar with the participant's information. Then, translated into English language transcripts by those investigators, compared, and argued for discrepancies. The investigators read and re-read the transcripts to familiarize themselves with the idea and developed memos and codes line by line against the themes and sub-themes of the framework, refined and compared with themes for emerged new themes and sub-themes. The investigators discussed until agreement regarding the inconsistencies, new ideas of the participants, emerged themes and sub-themes on daily bases, and the data collection continued until idea saturation. The data analyzed inductively by using a thematic content analysis approach in NVivo 12 Pro software.

\section{Results}


In this interview, sixteen-key informants were involved with mean age and standard deviation of $34.7 \pm 8$ years old. The majority ( $n=10$ ) participants were male, and 13 were an educational level of BSc (1st degree). Regarding work experience, 13 participants served five or more years in the health facility. Eleven participants were health professional qualifications of public health, and four were nurses. Overall, six main themes, twenty sub-themes, and fourteen sub-sub-themes were emerged in this study (Table 2).

Table 2: Main themes, sub-themes, and sub-sub-themes emerged in this study

\begin{tabular}{|c|c|}
\hline Main themes & Sub-themes \\
\hline \multicolumn{2}{|l|}{ Theme 1:Overview } \\
\hline \multirow[t]{2}{*}{ Theme 2:Consequences } & Stress on the HCPs \\
\hline & The socio-cultural and economic crisis \\
\hline \multirow[t]{4}{*}{ Theme 3:Perceived practice of preventive measures } & Using PPEs \\
\hline & Handwashing and using alcohol and sanitizer \\
\hline & Avoid contact \\
\hline & Appropriate waste disposal \\
\hline \multirow[t]{3}{*}{ Theme 5:Newfangled activities } & Launching testing center and other services \\
\hline & Controlling patients, visitors, and staffs \\
\hline & Taskforce organization and surveillance \\
\hline \multirow[t]{7}{*}{ Theme 6: Suggestions for improvement by HCPs } & Conduct outreach services and involve community leaders \\
\hline & Control transportation \\
\hline & Strengthen multidisciplinary teamwork \\
\hline & Motivation and capacity building for staffs \\
\hline & Fulfill necessary supplies \\
\hline & Monitor activities continuously \\
\hline & Mass screening and campaign \\
\hline
\end{tabular}

Continued... next to theme 3

\begin{tabular}{|c|c|c|}
\hline Main themes & Sub-themes & Sub-sub-themes \\
\hline \multirow{9}{*}{ Theme 4:Perceived militating factors } & \multirow{3}{*}{ Community influence } & Negligence and ignorance \\
\hline & & Inconsistent application of a facemask \\
\hline & & Miss conception about the disease \\
\hline & Health care provider related factors & Negligence and ignorance \\
\hline & \multirow[t]{2}{*}{ Institutional/facility-related factors } & Shortage of PPEs and solutions for hand hygiene \\
\hline & & Lack of infrastructure \\
\hline & \multirow[t]{3}{*}{ Lack of communication and support } & Lack of attention and recognition for staffs \\
\hline & & Lack of communication \\
\hline & & Lack of support from community and government \\
\hline
\end{tabular}




\section{Overview of COVID-19}

The majority of participants stated that the origin of COVID-19 was from the markets of Wuhan province, China, after eating animal meat (bat). It caused by a virus and the disease named by year of occurrence, called Coronavirus disease-2019 (COVID-19).

“Coronavirus disease-2019 (COVID-19) originated from Wuhan province, China after eating bat meat" (Participant 10)

"The name of disease given by the virus name and year of occurrence, which called Coronavirus Disease-2019" (Participant 14)

The most commonly reported modes of transmission of the COVID-19 were physical contact, air and droplets, and body fluids. Dry cough, fever, the difficulty of breathing/shortness of breathing, sore throat, back pain, and sneezing were the reported symptoms by the study participants.

"The mode of transmissions are physical contact, air, droplets, handshaking, and different body fluids" (Participant 15)

"The sign and symptoms of COVID-19 are fever, the difficulty of breathing/shortness of breathing, and sore throat" (Participant 16)

"The sign and symptoms of COVID-19 are fever, back pain, sneezing, and dry cough" (Participant 12)

The majority of the participants responded that homestay (social distancing), physical distancing, avoiding public gathering, hand washing, applying facemask, and using hand sanitizer or alcohol could prevent the transmission of the COVID-19. There is no proven drug or vaccine for the COVID-19 until now. Nevertheless, some of the participants stated that antibiotics used as treatment and taking cooked hot foods and fluids recommended as supportive management.

"There is no vaccine and treatment for Coronavirus Disease-2019. But, the best prevention way is homestay; others are not got to a public gathering, physical distancing, washing hands with soap and water, using alcohol or sanitizer, and using facemask at market or place where so many individuals gathered". Besides, to above-stated prevention ways, social or physical distancing at least 2 meters or $6 f t$. Reducing transport, not got to "Lekiso" or burial places, "Ikub" and Edir" places" (Participant 4)

"The prevention ways are social distancing, frequent washing with water and soap by rubbing hands for 20 seconds, using alcohol and sanitizer in areas where water is not available. Two staffs from our sectors trained on COVID-19 prevention and treatment and they stated that we could treat COVID-19 by antibiotics by increasing the dose (at least by Amoxicillin) and by taking cooked hot food and fluids"(Participant 3)

\section{Consequences of COVID-19 pandemic}

Tension on health professionals and absence from work, socio-cultural and economic crisis were the consequences of the COVID-19 pandemic. Currently, it is the utmost public health issue all over the world.

"COVID-19 is the most severe and resulted in tension on HCPs and economic crisis. It is possible to control the transmission but challenged with the societal culture and the way of living" (Participant 7)

\section{The perceived practice of preventive measures of the COVID-19 pandemic in health professionals}

The majority of the health professionals prevented themselves and others who came to the health facility by using personal protective equipment (PPEs) (facemask, glove, and gown), frequently washing hands with soap and water. They continuously maintained recommended physical distance as needed, distance beds in the ward, controlling the number of visitors, and order the visitors to use a facemask. All most all the health professionals use hand sanitizer or alcohol after any contact with patients. They identify and isolate patients with cough and high fever start from main get or pre-triage, and by practicing other infection prevention practices (IPPs) such as appropriate waste disposal system in the health facility.

"Health care professional prevent themselves and patients or any person who visits the health facility by using different kinds of masks (surgical mask, $\mathrm{N}$-95 facemask, and locally produced masks), frequently washing hands with soap and water, and by using hand sanitizers" (Participant 1)

"Health care workers, preventing themselves and others by using a facemask, which produced locally, and some bought their N-95 facemask and hand sanitizer. They wash hands with soap and water in the main get and emergency room only due to a shortage of handwashing facilities. The entrance of patients and visitors controlled in the main get. One person or supporter for severely ill patients and only patient if stand-alone can get into the facility. All individuals who enter the facility must apply a facemask and wash hand in the main get"(Participant 8)

"In the case of the pediatric ward, the patient came after passing pre-triage, and all the staff practice standard precautionary measures such as using a facemask, donning gloves, frequently washing hands, and use sanitizers. If the patients with cough came to the ward, there is isolation 
room until diagnosis and if we suspected COVID-19 sample sent to the testing center" (Participant 11)

"All health professionals have a facemask, and maintain physical distancing. However, it is difficult in case of maternal, child health services, family planning, delivery, and postnatal care because there is direct physical contact" (Participant 7)

"The health care workers preventing the pandemic by infection prevention practice such as avoiding or stored contaminated materials in specified places and follow the adequate waste disposal mechanisms, using PPEs (facemask, gloves, and gowns)" (Participant 15)

\section{Perceived militating factors for the practice of preventive measures for COVID-19}

\section{Community influence}

The main factor that affects the health care providers' practice of preventive measures for the COVID-19 pandemic was hampered by the lack of awareness of the community, negligence, and ignorance, not using a facemask and using incorrectly, and miss conception about the disease.

"There is ignorance and negligence in the community to accept the practice of prevention ways or to avoid the previous culture while came to our facility" (Participant 13)

"Most community members who came to institution perceived that there are no any COVID-19 and it is false and politics, not a disease. It is a common cold, which cured easily. Because no one is infected and no one is dead in our surrounding"(Participant 3)

"There is a lack of awareness in the community who came to the institution even if awareness created by different stakeholders by using montarbo and microphones. They came without a facemask, and some apply facemask inappropriately"(Participant 7)

\section{Health care provider related factors}

In contrast to the national response on COVID-19 prevention in a government organization that pregnant and breastfeeding staff, advanced age staff, and staff with chronic diseases were allowed to stay at home. However, in health care institutions, they are obligated to do work. Therefore, this may affect the willingness and motivation of the staff.

"The main challenge in this facility to practice precautionary measures in line with standards and emergency operation is that pregnant and breastfeeding staffs, aged staffs, and staffs with chronic diseases like asthma and diabetes mellitus were obligated to work and not allowed to stay at home" (Participant 3)

As reported by the utmost participants, negligence and ignorance were the most speculated factor or challenges in the practice of preventive measures for the COVID-19 pandemic.

"Regarding the practice precautionary measures to prevent COVID-19, most staffs practice the measures even if there are some negligent and ignorant staffs due to no occurrence of the case around the town" (Participant 16)

\section{Institutional/ facility-related factors}

he shortage of PPEs (facemask, and glove), scarcity of hand cleaning solutions (alcohol, and sanitizer), shortage of training and trained staffs were the most common militating factors that influence the practice of preventive measures. Similarly, unavailability of guidelines, water shortage, inadequate duty, OPD, and emergency rooms were also point outed by key informants.

"The militating factors are the absence of hand sanitizer, shortage of alcohol, facemasks, and other PPE since our district is new, and the majority of the thinks are limited. There is no screening in our institution as well as in our district even with infrared thermometer" (Participant 5)

"The militating factors that affect the practice of precautionary measures in our institution are..., shortage of supplies (facemask, gloves, etc.), inadequate OPD and emergency rooms to maintain the recommended physical distance. The other factors are...unavailability of guidelines to orient the staffs" (Participant 15)

"There is no training and psychological support for HCP to avoid stress and to stabilize the situation. In general, the budget issue main concern to fulfill the necessary materials and to train all the health care providers. As our health facility, there is no water, we buy 20 litter/ 10 birr per day water from surrounding, and we use rainwater, which strode in the tanker, there is also a shortage of alcohol and sanitizer, and district health office gave two litter alcohol and sanitizer" (Participant 4) 
"The militating factors that affect the practice of precautionary measures in our institution are inadequate duty rooms for staffs that many staffs exchangeable used a single room for a period and if one individual from those infected the risk contamination is most likely. The other challenge is the shortage of hand sanitizer and facemask. The recommended duration of use of single facemask is 8 hour, but most staffs use more than a week" (Participant 6)

"The main problem in this health facility is that as different teams or stakeholders, commented there is no triage. However, we simply isolated patients with cough and gave priority for those individuals" (Participant 3)

\section{Lack of communication and support}

Some of the participants highlighted that lack of communication, support from the community and government, and lack of attention and recognition for staff were factors that influence the practice of precautionary measures for the COVID-19 pandemic in the health care facility.

"The militating factors that affect the practice of precautionary measures are attention and recognition not given for health care professionals... There is no support from the town health office, zonal health department, and task force in the zone rather than taking the suspected or confirmed cases to the treatment center. Staffs in other government organization apply standard and recommended facemask, but we struggled to serve the community by locally produced and below standard facemasks"(Participant 8)

"The other factors are lack of communication of our institution with supporting organization or universities to train the staffs and unavailability of guidelines to orient the staffs"(Participant 15)

\section{Newfangled activities in the health facility after the occurrence of the pandemic}

Organization of fever clinic and pre-triage service, hand washing station, and screening with infrared thermometer or thermo-scan in the get were the major newfangled activities after the occurrence of the COVID-19 pandemic. Individuals are also controlled and obligated to apply facemask while entering the health facility.

"Activities that conducted after the occurrence of the pandemic are an organization of fever clinic that provided by trained staffs with an infrared thermometer, pre-triage service, and COVID-19 testing center launched. Handwashing facilities organized in a different place and all persons are obligated to apply facemask" (Participant 12)

"The activities conducted in the facility after the occurrence of the pandemic were a team (taskforce) created and a team of health professionals trained in the zonal level. There is integration with health extension workers at the health post, and those health extension workers conducted surveillance at least 20 households per day to identify the suspected cases"(Participant 3)

"The activities conducted in our facility after the pandemic was the construction of new handwashing station, screening in the get, distancing patient bed in the ward, reducing the number participates in meeting"(Participant 2)

\section{Suggestions by health professionals for the improvement}

Involving and integrating the awareness creation activities with the community leaders, strengthening and monitoring the activities of different task forces, extensive training for health professionals and leaders in the health facility were the issues suggested by the participants. Surveillance with mass screening, outreach services to create awareness in the community, transportation control, and attention and recognition for health professionals were also points recommended.

"...the community leader must intervene on this to save the community from this pandemic. The community practicing already an existing culture in "Eder" "Ekub" and "Lekiso" places, that the task force, the town health office, and the zonal health department must support the health professionals or team to create awareness in the community..."(Participant 8)

"...awareness creation must be continued in the community... transport access must arrange...for the staffs who came far from the health facility" (Participant 7)

"...task force activities are key roles to prevent the spread of infection in the community. Therefore, those activities must strengthen and frequently monitored rather than one session or one-week activity" (Participant 1)

"...the leaders in the facility must train on COVID-19 prevention aspects before anyone that the leaders are the main responsible person or individual to orient or to facilitate the health professionals or staffs to practice prevention measures"(Participant 2)

"...mass screening needed to identify the case, staffs and all the community must apply a facemask and use hand sanitizer..."(Participant 12) 


\section{Discussion}

\section{Summary}

The novel coronavirus is a global medical pandemic that resulted in stress on the health care system. There is no proven drug and vaccines for this disease. Because of the high contagiousness, strictly practicing precautionary measures can save life many populations, including health care providers. Studies are emerging related to the epidemiological aspects, social, political, and economic consequences of the COVID-19. Nevertheless, few are exploring militating factors. As such, this aimed to fill this research gap in country Ethiopia. The study found that negligence and ignorance, shortage of PPEs, lack of infrastructure, lack of training, lack of communication, and lack of attention and recognition for staff were the most common militating factors for the practice of precautionary measures for COVID-19 in the health facility.

\section{Comparison and contrast with exiting evidence}

Less commitment of health care workers to the policies and procedures was one of the factors that the health professionals did not practice prevention ways and effected with the COVID-19 $[28,29]$. This study also reconnoitered that negligence and ignorance among health care workers were the most speculated factor or challenges in the practice of preventive measures for the COVID-19 pandemic. The reason is that most people, including health professionals, have miss conception about the disease and the contagiousness, and due to that case, they do not give attention to the prevention practices and abandon due to not the occurrence of the disease in the surrounding that they reside.

This study found that a shortage of PPEs (facemasks, and glove) and scarcity of hand cleaning solutions (alcohol, and sanitizer) had the main militating factors. These findings also are shown in studies conducted elsewhere [28-35]. These are evidence that if the health professionals do not get enough amount of PPEs, they are obligated to do the procedures or come in contact with patients with available resources, and a breach in standard precautions inevitable. These resulted in the health professionals more vulnerable to the infection and more likely to transmit the disease (COVID-19) to others. Studies conducted in Ethiopia [32], Pakistan [28], and China [31] reported that lack of access to infection prevention training or insufficient/inadequate training in infection control measures resulted in the poor practice of precautionary measures for the COVID-19. A result of systematic review also supplements the findings of the above-stated literature. Health professionals not trained on the infection and about how to use PPEs, and they reported it was problem when training was not mandatory [34]. The other study stated institutional (training and education, policies, and management expectations) factors are essential to improve the practice of preventive measures [35]. In line with this, this study found that a shortage of training for the staff (health professionals) had one of the militating factors that affect the practice of precautionary measures for COVID-19. Provide training for the health professionals on overall aspects of the COVID19 , including precautionary measures in the health care facility, can improve the practice of preventive measures.

Evidence showed that tied to the guideline itself and how it communicated, support from managers, workplace culture, lack of physical space, and poor design of the hospitals were influence the health care workers ability and willingness to follow IPC guidelines and infection prevention practice when managing respiratory infectious diseases [32,34,36]. This study also identified that lack of communication and support, unavailability of the guidelines, and shortage of water supply, inadequate duty, OPD, and emergency rooms were factors influencing the practice of preventive measures. The reason is that updated guidelines, communication with the different supporting teams and support from concerned bodies, adequate facilities to provide quality services with the standard are pivotal to practice precautionary measure for COVID-19 in the health facility.

Lack of attention and recognition for staffs, lack of motivation of the health professionals and obligated to do work, and community influence (lack of awareness, negligence and ignorance, not using facemask and using incorrectly, and miss conception about the disease) were also pointed out in this study that it hampered the practice of preventive measures for COVID-19. These are facts that give attention and recognition for those in the front line, the motivation of the staff by fulfilling the thing that they urgently need as much as possible, and community vigilance can improve the practice.

\section{Limitation}

Only two interviewers who has an expert on qualitative data collection and who have full information and idea about the study protocol were involved to ensure consistency and bracketing. However, due to the nature of the disease transmission and the state emergency declaration, the ethical review board was not allowed to do focus group discussion. The sample size of this study relatively small, and only leader (key informants) were involved. These may affect the transferability of the study results.

\section{Implications}

This study can be input for the policymakers and program evaluators to design appropriate strategies for training, capacity building, and other activities for service providers and immediate intervention in health institution based on the identified gaps. The study has shown the foremost measurements on the perceived militating factors for the practice of preventive measures, and this ultimately benefits the health care workers, 
managers, and leaders at each level in the health care system and other stakeholders. The finding also serves as a tool for public policy and case management teams to evaluate the prevention and control mechanism of the novel coronavirus in the health care system. Besides, it also provides the basic framework for future studies in assessing and comparing the performance of interventions.

\section{Conclusions}

This study found that there was a gap in practicing preventive measures for COVID-19 in health facilities among health professionals due to multiple of militating factors. Shortage of PPEs and hand cleaning solution, shortage of training, negligence and ignorance, and poor infrastructure in the facility were the most common militating factors that need immediate attention. Therefore, the government, the ministry of health, non-governmental organizations, and other voluntary supports should fulfill the necessary supplies in the health facilities, improve the infrastructures, equip health professionals by providing capacity-building activities. Besides, attention and recognition must give for the health care professionals, and credit should consider.

\section{List Of Abbreviations}

COVID-19: Coronavirus Diseases-2019, HCPs: Health Care Professionals, HCWs: Health Care Worker's, KII: Key Informant Interview, OPD: Outpatient Department, and PPEs: Personal Protective Equipment's

\section{Declarations}

\section{Ethics approval and consent to participate}

Institutional Research Ethics Review Board (IRB) of the Arba Minch University, College of Medicine, and Health Sciences provided the ethical clearance. The reference number of the letter was IRB/408/12. Written and signed voluntary consent obtained from all the key informants. The concealment kept via the use of codes. The key informants also informed that the information obtained from them kept with the utmost confidentiality.

\section{Consent for publication}

Not applicable

\section{Availability of data and materials}

The data (audios and transcripts) will not be shared to preserve participant anonymity.

\section{Competing interests}

All authors assert that they have no competing interests

\section{Funding}

Arba Minch University provided funds for this research work with a project grant code of GOV/AMU/COVID-

19/PDNPI/CMHS/RCSVPO/012/2020. The website of the university is www.amu.edu.et. "The funders had no role in study design, data collection, and analysis, decision to publish, or preparation of the manuscript."

\section{Authors' contributions}

AM designed the study, involved in data collection, in data analysis, interpretation of the result, and drafted the paper, and participated in preparing all versions of the manuscript. SS, MG, GA, AB, MK, SA, and MS assisted in the design and the proposal development, monitored data collection, assisted during analysis, and revised subsequent drafts of the paper. All authors read and approved the final manuscript.

\section{Acknowledgments}

The authors are glad to forward their appreciation to Arba Minch University for offering financial support to take on this research project. We would also like to extend our heartfelt gratitude to the Health Departments, CEOs, Medical directors of hospitals, and head of health centers for giving information and baseline data about the study area and key informants in each health facility as well as helping during data collection. Last but not least, we are indebted to the endeavor made by interviewers to expedite this research process.

\section{Authors' information}


Abera Mersha (BScN, MSc in Maternity and Neonatal Nursing), School of Nursing, College of Medicine and Health Sciences, Arba Minch University, Arba Minch, Ethiopia.

Shitaye Shibiru (BScN, MSc in Maternity and Reproductive Health Nursing), School of Nursing, College of Medicine and Health Sciences, Arba Minch University, Arba Minch, Ethiopia.

Meseret Girma (MPH, Assistant Professor of General Public Health), School of Public Health, College of Medicine and Health Sciences, Arba Minch University, Arba Minch, Ethiopia.

Gistane Ayele (MPH, Assistant Professor of Monitoring and Evaluation), School of Public Health, College of Medicine and Health Sciences, Arba Minch University, Arba Minch, Ethiopia.

Agegnehu Bante (BScN, MSc in Maternity and Neonatal Nursing), School of Nursing, College of Medicine and Health Sciences, Arba Minch University, Arba Minch, Ethiopia.

Mekidim Kassa (BScN, MPH in Biostatistics and Epidemiology), School of Public Health, College of Medicine and Health Sciences, Arba Minch University, Arba Minch, Ethiopia.

Sintayehu Abebe (BScMw, MPH in Reproductive Health), School of Public Health, College of Medicine and Health Sciences, Arba Minch University, Arba Minch, Ethiopia.

Misgun Shewangizaw (MPH, Assistant Professor of Health Education and Promotion), School of Public Health, College of Medicine and Health Sciences, Arba Minch University, Arba Minch, Ethiopia.

\section{References}

1. WHO. WHO Statement Regarding Cluster of Pneumonia Cases in Wuhan, China, 2020. Available at: https://www.who.int/china/news/detail/09-01-2020-who-statement-regarding-cluster-of-pneumonia-cases-in-wuhan-china. Access date: [April 27, 2020].

2. Xinhua News Agency, Beijing. Experts say that the pathogenic identification of the novel coronavirus of unknown cause in Wuhan has made preliminary progress. Available at: http://www.xinhuanet.com/2020-01/09/c_1125438971.htm. Access date: [April 27, 2020].

3. Holmes E. Novel-2019 Coronavirus Genome. Available at: http://virological.org/t/novel-2019-coronavirus-genome/319. Access date: [April 27, 2020].

4. GenBank. Severe acute respiratory syndrome coronavirus 2 isolate Wuhan-Hu-1, complete genome. Available at: https://www.ncbi.nIm.nih.gov/nuccore/MN908947. Access date: [April 27, 2020].

5. World Health Organization (WHO). Coronavirus disease 2019 (COVID-19)Situation Report -10, 2020.

6. Wuhan City Health Committee (WCHC). Wuhan Municipal Health and Health Commission's briefing on the current pneumonia epidemic situation in our city, 2019. Available at: http://wjw.wuhan.gov.cn/front/web/showDetail/2019123108989. Access date: [April 26, 2020].

7. National Health Commission of the People's Republic of China. Prevent guidelines of 2019-nCoV, 2020. Available at: http://www.nhc.gov.cn/xcs/yqfkdt/202001/bc661e49b5bc487dba182f5c49ac445b.shtml. Access date: [April 27, 2020].

8. National Health Commission of the People's Republic of China. Pneumonia diagnosis and treatment of 2019-nCoV infection from Chinese $\mathrm{NHC}$ and CDC 2020, 2020. Available at:

http://www.nhc.gov.cn/xcs/zhengcwj/202001/4294563ed35b43209b31739bd0785e67/files/7a9309111267475a99d4306962c8bf78.pdf. Access date: [April 27, 2020].

9. CDC. 2019-Novel coronavirus, Wuhan, China. 2020. Available at: https://www.cdc.gov/coronavirus/2019-nCoV/summary.html. Access date: [April 27, 2020].

10. Huang C, Wang Y, Li X, Ren L, Zhao Jianping, Hu Y, et al. Clinical features of patients infected with 2019 novel coronavirus in Wuhan, China. Lancet. 2020; 395:497-506. https://doi.org/10.1016/S0140-6736(20)30183-5.

11. Adhikari SP, Meng S, Wu YJ, Mao YP, Ye RX, Wang QZ, et al. Epidemiology, causes, clinical manifestation and diagnosis, prevention and control of coronavirus disease (COVID-19) during the early outbreak period: a scoping review. Infectious Diseases of Poverty, 2020.9 (1):29.

12. Li T, Wei C, Li W, Hongwei F, Shi J. Beijing Union Medical College Hospital on "pneumonia of novel coronavirus infection" diagnosis and treatment proposal (V2.0). Med J Peking Union Med Coll Hosp., 2020.

Available at: http://kns.cnki.net/kcms/detail/11.5882.r.20200130.1430.002.html. Access date: [April 27, 2020]. 
13. The medical expert group of Tongji hospital. A quick guide to the diagnosis and treatment of pneumonia for novel coronavirus infections (third edition). Herald Med. 2020. Available at: http://kns.cnki.net/kcms/detail/42.1293.r.20200130.1803.002.html. Access date: [April 27, 2020].

14. WHO. Coronavirus disease (COVID-19) outbreak: rights, roles, and responsibilities of health workers, including key considerations for occupational safety and health, 2020. Available at: https://www.who.int/publications-detail/coronavirus-disease-(covid-19)-outbreakrights-roles-and-responsibilities-of-health-workers-including-key-considerations-for-occupational-safety-and-health. Access date: [May 2, 2020].

15. Hoe GW, Wah LJ, Koh D. Preventing intra-hospital infection and transmission of COVID-19 in health care workers. Safety and health at work, 2020: 10.1016/j.shaw.2020.03.001.

16. Nemati M, Ebrahimi B, Nemati F. Assessment of Iranian Nurses' Knowledge and Anxiety Toward COVID-19 During the Current Outbreak in Iran. Arch Clin Infect Dis 2020; In Press. https://doi.org/10.5812/archcid.102848.

17. National Institute for Communicable Disease. Clinical Management of Suspected or Confirmed COVID-19 Diseases, 2020. Available at: https://www.nicd.ac.za/wp-content/uploads/2020/03/Clinical-Management-of-COVID-19-disease_Version-3_27March2020.pdf. Access date: [May 2, 2020].

18. WHO. Advice on the use of masks in the community, during home care, and in health care settings in the context of the novel coronavirus 2019-nCoV outbreak (Interim guidance). 2020. Available at: WHO/nCov/IPC_Masks/2020. Access date: [April 27, 2020].

19. USAID, PEPFAR. Strategic Considerations for Mitigating the Impact of COVID-19 on Key-Population-Focused HIV Programs, 2020. Available at: https://www.fhi360.org/sites/default/files/media/documents/epic-kp-strategic-considerations-covid-19.pdf. Access date: [April 28, 2020].

20. McKibbin WJ and Fernando R. The Global Macroeconomic Impacts of COVID-19: Seven Scenarios, 2020. CAMA Working Paper No. 19/2020. Available at SSRN: https://ssrn.com/abstract=3547729 or http://dx.doi.org/10.2139/ssrn.3547729

21. World Health Organization (WHO). Coronavirus disease-2019 (COVID-19)Situation Report -97, 2020.

22. WHO. WHO urges countries not to let COVID-19 eclipse other health issues, 2020. Available at: https://www.afro.who.int/news/who-urgescountries-not-let-covid-19-eclipse-other-health-issues. Access date:[April 27, 2020].

23. Liu Q, Luo D, Haase JE, Guo Q, Wang XQ, Liu S, Xia L, Liu Z, Yang J, Yang BX. The experiences of health-care providers during the COVID-19 crisis in China: a qualitative study, Lancet Glob Health 2020. https://doi.org/10.1016/S2214-109X(20)30204-7.

24. WHO. Strengthening the Health Systems Response to COVID-19. Version April 2020Strengthening the Health Systems Response to COVID19 Technical working guidance \#1 Maintaining the delivery of essential health care services freeing up resources for the COVID-19 response while mobilizing the health workforce for the COVID-19 response (18 April 2020). Available at: http://www.euro.who.int/_data/assets/pdf_file/0007/436354/strengthening-health-systems-response-COVID-19-technical-guidance-1.pdf. Access date: [May 2. 2020].

25. UpToDate. Coronavirus disease 2019 (COVID-19): Infection control in health care and home settings. Available at: https://www.uptodate.com/contents/coronavirus-disease-2019-covid-19-infection-control-in-health-care-and-home-settings. Access date: [May 2, 2020].

26. Khader Y, Al Nsour M, Al-Batayneh OB, Saadeh R, Bashier H, Alfaqih M, Al-Azzam S, AlShurman BA Dentists' Awareness, Perception, and Attitude Regarding COVID-19 and Infection Control: Cross-Sectional Study Among Jordanian Dentists. JMIR Public Health Surveill, 2020;6(2):e18798.

27. Kumar J, Katto M, Siddiqui A A, et al. Knowledge, Attitude, and Practices of Healthcare Workers Regarding the Use of Face Mask to Limit the Spread of the New Coronavirus Disease (COVID-19). Cureus, April 20, 2020; 12(4): e7737. DOI:10.7759/cureus.7737

28. Saqlain M, Munir MM, Rehman SU, Gulzar A, Naz S, Ahmed Z, et al., Knowledge, Attitude and Practice among Healthcare Professionals regarding COVID-19: A cross-sectional survey from Pakistan, 2020.

29. Maleki S, Naja F, Farhadi K, Fakhri M, Hosseini F, Naderi M. Knowledge, Attitude, and Behavior of Health Care Workers in the Prevention of COVID-19, 2020.

30. Abdel WY, Hefzy EM, Ahmed MI, Hamed NS. Assessment of Knowledge, Attitudes, and Perception of Health Care Workers Regarding COVID19, A Cross-Sectional Study from Egypt. Journal of community health, 2020:1-10.

31. Wang J, Zhou M, Liu F. Reasons for health care workers becoming infected with novel coronavirus disease 2019 (COVID-19) in China. The Journal of hospital infection, 2020. 105(1):100-101.

32. Asemahagn MA. Factors determining the knowledge and prevention practice of health care workers towards COVID-19 in Amhara region, Ethiopia: a cross-sectional survey. Tropical Medicine and Health, 2020. 48(1):72.

33. Ayinde O, Usman AB, Aduroja P, Gbolahan A. A cross-sectional study on Oyo state, health care workers knowledge, attitude and practice regarding coronavirus disease 2019 (COVID-19); 2020. 
34. Houghton C, Meskell P, Delaney H, Smalle M, Glenton C, Booth A, Chan XHS, Devane D, Biesty LM. Barriers and facilitators to health care workers' adherence to infection prevention and control (IPC) guidelines for respiratory infectious diseases: a rapid qualitative evidence synthesis. Cochrane Database of Systematic Reviews 2020, Issue 4. Art. No.: CD013582. DOI: 10.1002/14651858.CD013582.

35. Cooper S, Wiyeh A, Schmidt BM, Wiysonge CS. Cochrane corner: factors that influence compliance by health care workers with infection prevention and control guidelines for COVID-19 and other respiratory infections. Pan Africa Medical Journal. 2020;35(2):23. DOI: 10.11604/pamj.2020.35.2.23012.

36. Ughasoro MD, Esangbedo DO, Udorah IM. Health Care Workers' Perspectives on Preparedness of Health Care Facilities for Outbreak of Communicable Diseases in Nigeria: A Qualitative Study. Am. J. Trop. Med. Hyg., 100(4), 2019, pp. 1022-1028, DOI:10.4269/ajtmh.180404.

\section{Supplementary Files}

This is a list of supplementary files associated with this preprint. Click to download.

- Supplementaryfile1.pdf 